\title{
BFKL Effects in Azimuthal Angle Correlations of Forward Jets
}

\author{
Agustín Sabio Vera $^{1}$ and Florian Schwennsen ${ }^{2} *$ \\ 1- Physics Department - Theory Division CERN \\ CH-1211 Geneva 23 - Switzerland \\ 2- II. Institut für Theoretische Physik - Universität Hamburg \\ Luruper Chaussee 149 D-22761 Hamburg - Germany
}

\begin{abstract}
The azimuthal angle correlation of Mueller-Navelet jets at hadron colliders is studied in the NLO BFKL formalism. We highlight the need of collinear improvements in the kernel to obtain good convergence properties and we obtain better fits for the Tevatron data than at LO accuracy. We also estimate these correlations for larger rapidity differences available at the LHC.
\end{abstract}

\section{BFKL cross sections}

In [2] we continue the study initiated in [3] of azimuthal correlations in Mueller-Navelet jets [4] using the Balitsky-Fadin-Kuraev-Lipatov (BFKL) equation in the next-to-leading (NLO) approximation [5]. We investigate normalized differential cross sections which are quite insensitive to parton distribution functions and read

$$
\frac{d \hat{\sigma}}{d^{2} \vec{q}_{1} d^{2} \vec{q}_{2}}=\frac{\pi^{2} \bar{\alpha}_{s}^{2}}{2} \frac{1}{q_{1}^{2} q_{2}^{2}} \int \frac{d \omega}{2 \pi i} e^{\omega \mathrm{Y}} f_{\omega}\left(\vec{q}_{1}, \vec{q}_{2}\right),
$$

where $\bar{\alpha}_{s}=\alpha_{s} N_{c} / \pi, \vec{q}_{1,2}$ are the transverse momenta of the tagged jets, and Y their relative rapidity. The Green's function carries the Y-dependence and follows the NLO equation, $\left(\omega-\bar{\alpha}_{s} \hat{K}_{0}-\bar{\alpha}_{s}^{2} \hat{K}_{1}\right) \hat{f}_{\omega}=\hat{1}$, which acts on the basis including the azimuthal angle, $\langle\vec{q} \mid \nu, n\rangle=\frac{1}{\pi \sqrt{2}}\left(q^{2}\right)^{i \nu-\frac{1}{2}} e^{i n \theta}$. As Y increases the azimuthal dependence is driven by the kernel. This is why we use the LO jet vertices which are simpler than at NLO. The differential cross section in the azimuthal angle $\phi=\theta_{1}-\theta_{2}-\pi$, with $\theta_{i}$ being the angles of the two tagged jets, is

$$
\begin{aligned}
\frac{d \hat{\sigma}\left(\alpha_{s}, \mathrm{Y}, p_{1,2}^{2}\right)}{d \phi} & =\frac{\pi^{2} \bar{\alpha}_{s}^{2}}{4 \sqrt{p_{1}^{2} p_{2}^{2}}} \sum_{n=-\infty}^{\infty} e^{i n \phi} \mathcal{C}_{n}(\mathrm{Y}) \\
\mathcal{C}_{n}(\mathrm{Y}) & =\frac{1}{2 \pi} \int_{-\infty}^{\infty} \frac{d \nu}{\left(\frac{1}{4}+\nu^{2}\right)}\left(\frac{p_{1}^{2}}{p_{2}^{2}}\right)^{i \nu} e^{\chi\left(|n|, \frac{1}{2}+i \nu, \bar{\alpha}_{s}\left(p_{1} p_{2}\right)\right) \mathrm{Y}} \\
\chi\left(n, \gamma, \bar{\alpha}_{s}\right) & =\bar{\alpha}_{s} \chi_{0}(n, \gamma)+\bar{\alpha}_{s}^{2}\left(\chi_{1}(n, \gamma)-\frac{\beta_{0}}{8 N_{c}} \frac{\chi_{0}(n, \gamma)}{\gamma(1-\gamma)}\right) .
\end{aligned}
$$

The eigenvalue of the LO kernel is $\chi_{0}(n, \gamma)=2 \psi(1)-\psi\left(\gamma+\frac{n}{2}\right)-\psi\left(1-\gamma+\frac{n}{2}\right)$, with $\psi$ the logarithmic derivative of the Euler function. The action of $\hat{K}_{1}$, in $\overline{\mathrm{MS}}$ scheme, can be found

*Supported by the Graduiertenkolleg "Zukünftige Entwicklungen in der Teilchenphysik" 
in [6]. The full cross section only depends on the $n=0$ component, $\hat{\sigma}=\frac{\pi^{3} \bar{\alpha}_{s}^{2}}{2 \sqrt{p_{1}^{2} p_{2}^{2}}} \mathcal{C}_{0}(\mathrm{Y})$. The average of the cosine of the azimuthal angle times an integer projects out the contribution from each of these angular components:

$$
\frac{\langle\cos (m \phi)\rangle}{\langle\cos (n \phi)\rangle}=\frac{\mathcal{C}_{m}(\mathrm{Y})}{\mathcal{C}_{n}(\mathrm{Y})}
$$

The normalized differential cross section is

$$
\frac{1}{\hat{\sigma}} \frac{d \hat{\sigma}}{d \phi}=\frac{1}{2 \pi} \sum_{n=-\infty}^{\infty} e^{i n \phi} \frac{\mathcal{C}_{n}(\mathrm{Y})}{\mathcal{C}_{0}(\mathrm{Y})}=\frac{1}{2 \pi}\left\{1+2 \sum_{n=1}^{\infty} \cos (n \phi)\langle\cos (n \phi)\rangle\right\} .
$$

The BFKL resummation is not stable at NLO [7, 8]. In the gluon-bremsstrahlung scheme our distributions become unphysical. To improve the convergence we impose compatibility with renormalization group evolution in the DIS limit [9] for all angular components. A good scheme is the angular extension of that discussed in [8], first proposed in [7]:

$$
\begin{aligned}
& \omega=\bar{\alpha}_{s}\left(1+\mathcal{A}_{n} \bar{\alpha}_{s}\right)\left\{2 \psi(1)-\psi\left(\gamma+\frac{|n|}{2}+\frac{\omega}{2}+\mathcal{B}_{n} \bar{\alpha}_{s}\right)\right. \\
& \left.-\psi\left(1-\gamma+\frac{|n|}{2}+\frac{\omega}{2}+\mathcal{B}_{n} \bar{\alpha}_{s}\right)\right\}+\bar{\alpha}_{s}^{2}\left\{\chi_{1}(|n|, \gamma)-\frac{\beta_{0}}{8 N_{c}} \frac{\chi_{0}(n, \gamma)}{\gamma(1-\gamma)}\right. \\
& \left.\left.-\mathcal{A}_{n} \chi_{0}(|n|, \gamma)\right)+\left(\psi^{\prime}\left(\gamma+\frac{|n|}{2}\right)+\psi^{\prime}\left(1-\gamma+\frac{|n|}{2}\right)\right)\left(\frac{\chi_{0}(|n|, \gamma)}{2}+\mathcal{B}_{n}\right)\right\},
\end{aligned}
$$

where $\mathcal{A}_{n}$ and $\mathcal{B}_{n}$ are collinear coefficients. After this collinear resummation our observables have a good physical behavior and are independent of the renormalization scheme.

\section{Phenomenology}

The $\mathrm{D} \emptyset[11]$ collaboration analyzed data for Mueller-Navelet jets at $\sqrt{s}=630$ and 1800 $\mathrm{GeV}$. For the angular correlation LO BFKL predictions were first obtained in [12, 13] and failed to describe the data estimating too much decorrelation. An exact fixed NLO analysis using JETRAD underestimated the decorrelation, while HERWIG was in agreement with the data.

In Fig. 2 we compare the Tevatron data for $\langle\cos \phi\rangle=\mathcal{C}_{1} / \mathcal{C}_{0}$ with our LO, NLO and resummed predictions. For Tevatron's cuts, where the transverse momentum for one jet is $20 \mathrm{GeV}$ and for the other $50 \mathrm{GeV}$, the NLO calculation is instable under renormalization scheme changes. The convergence of our observables is poor whenever the coefficient associated to zero conformal spin, $\mathcal{C}_{0}$, is involved. If we eliminate this coefficient by calculating the ratios defined in Eq. (1) then the predictions are very stable, see Fig. 2. The full angular dependence studied at the Tevatron by the $\mathrm{D} \emptyset$ collaboration was published in [11]. In Fig. 1 we compare this measurement with the predictions obtained in our approach. For the differential cross section we also make predictions for the LHC at larger Y in Fig. 3. Our calculation is not exact and we estimated several uncertainties, which are represented by gray bands. 

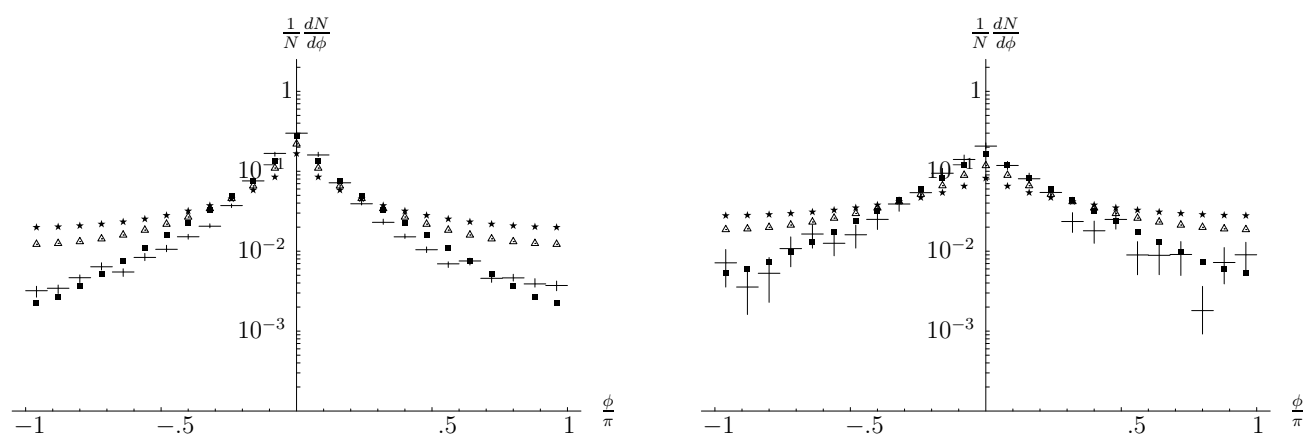

Figure 1: $\frac{1}{N} \frac{d N}{d \phi}$ in a $p \bar{p}$ collider at $\sqrt{s}=1.8 \mathrm{TeV}$ using a LO (stars), NLO (squares) and resummed (triangles) BFKL kernel. Plots are shown for $\mathrm{Y}=3$ (left) and $\mathrm{Y}=5$ (right).
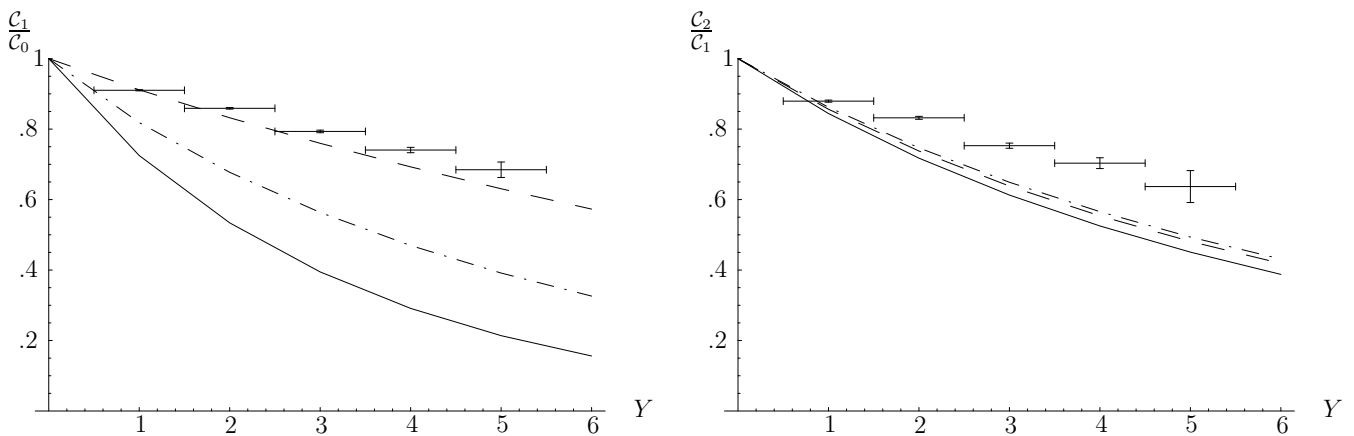

Figure 2: Left: $\langle\cos \phi\rangle=\mathcal{C}_{1} / \mathcal{C}_{0}$ and Right: $\frac{\langle\cos 2 \phi\rangle}{\langle\cos \phi\rangle}=\frac{\mathcal{C}_{2}}{\mathcal{C}_{1}}$, at a $p \bar{p}$ collider with $\sqrt{s}=1.8 \mathrm{TeV}$ for BFKL at LO (solid) and NLO (dashed). The results from the resummation presented in the text are shown as well (dash-dotted). 


\section{Conclusions}

We have presented an analytic study of NLO BFKL corrections in azimuthal angle decorrelations for Mueller-Navelet jets at hadron colliders. We found that the intercepts for non-zero conformal spins have good convergence. The zero conformal spin component needs of a collinear improvement to get stable results. Uncertainties in our study can be reduced using Monte Carlo techniques. We compared to the data extracted at the Tevatron many years ago. Our results improve with respect to the LO BFKL predictions but show too much azimuthal angle decorrelation. The LHC at CERN will have larger rapidity differences and will be a very useful tool to investigate the importance of BFKL effects in multijet production [15].

\section{References}

[1] Slides: http://indico. cern. ch/contributionDisplay . py? contribId=281\&sessionId=8\&conf Id=9499

[2] A. Sabio Vera, F. Schwennsen, Nucl. Phys. B 776 (2007) 170.

[3] A. Sabio Vera, Nucl. Phys. B 746 (2006) 1.

[4] A. H. Mueller, H. Navelet, Nucl. Phys. B 282 (1987) 727.

[5] V.S. Fadin, L.N. Lipatov, Phys. Lett. B 429, 127 (1998); G. Camici, M. Ciafaloni, Phys. Lett. B 430, 349 (1998).

[6] A. V. Kotikov, L. N. Lipatov, Nucl. Phys. B 582 (2000) 19.

[7] G. P. Salam, JHEP 9807, 019 (1998).

[8] A. Sabio Vera, Nucl. Phys. B 722 (2005) 65.

[9] M. Ciafaloni, D. Colferai, G. P. Salam, A. M. Stasto, Phys. Rev. D 68 (2003) 114003.

[10] B. Abbott et al., Phys. Rev. Lett. 84 (2000) 5722.

[11] S. Abachi et al., Phys. Rev. Lett. 77 (1996) 595.

[12] V. Del Duca, C. R. Schmidt, Phys. Rev. D 49 (1994) 4510.

[13] W. J. Stirling, Nucl. Phys. B 423 (1994) 56.

[14] C. L. Kim, FERMILAB-THESIS-1996-30.

[15] J. Bartels, A. Sabio Vera, F. Schwennsen, JHEP 0611 (2006) 051. 